\title{
Neue Materialflusskomponenten für die zukünftige Logistik
}

\author{
Prof. DR.-ING. KARL-HEINZ WEHKING \\ UNIVERSITÄT STUTTGART, INSTITUT FÜR FÖRDERTECHNIK UND LOGISTIK (IFT)
}

In den zurückliegenden 40 Jahren hat sich die Logistik anfänglich mit reinen Transportaufgaben befasst. Im Vergleich dazu geht es heute vornehmlich um Supply Chain-Systeme. Im Gegensatz zur klassischen Produktion entstehen hierbei allerdings keine Waren und Produkte, denn Logistik beschäftigt sich bekanntlich vorwiegend mit Dienstleistungen: von der Kundenbestellung über die Produktion bis zur Auslieferung. Und zwar innerhalb geschlossener Wertstoffkreisläufe, also auch unter Berücksichtigung der Entsorgung und Ressourcen sparender Rückeinspeisung.

Forty years ago logistics mainly dealt with transportation while nowadays its main focus lies on supply chain systems. In contrast to classical production, however, logistics provides no goods or products but services from the customer order over production up to the delivery. These services are provided within closed material cycles, i.e. under consideration of waste disposal and resource-saving recycling.

Sowohl in der Praxis als auch in der Theorie ist es unbestritten, dass die Logistik zurzeit eine Querschnittsfunktion in der Wirtschaft darstellt, sie ist somit ein wichtiges treibendes Element für die angestrebten Erfolge. Nach verschiedenen Prognosen ist davon auszugehen, dass der Markt für Logistik allein in Deutschland ein Umsatzvolumen von 150 bis 300 Mrd. Euro erreicht [Klaus].

Die Querschnittsfunktion der Logistik macht es notwendig, nicht etwa eine Optimierung von Einzelfunktionen durchzuführen. Vielmehr ist Logistik unter folgenden wesentlichen Gesichtspunkten ganzheitlich zu betrachten:

- Materialflusstechnik

- Informations- und Kommunikationstechnik

- betriebswirtschaftliche Beurteilung und Optimierung

In den letzten Jahren war festzustellen, dass Stichworte wie „IT-Technologie“ sowie „Optimierung der Ablaufund Aufbauorganisation“ innerhalb der Logistikbranche eine besondere Verbreitung und Bedeutung erlangt haben. Die technischen Bereiche waren im Vergleich dazu stark „unterbelichtet“.

\section{Paletten oder Container als Ladungsträger}

Wendet man sich der technischen Basis der Logistik zu, nämlich den Komponenten und Systemen des Materialflusses, dann lässt sich vereinfachend, aber mit deutlich ausgeprägtem Schwerpunkt feststellen, dass ein Großteil der heutigen Logistiksysteme und deren Optimierung aus technischer Sicht durch den Einsatz von Paletten oder Containern als Ladungsträger geprägt ist (Abb. 1). 

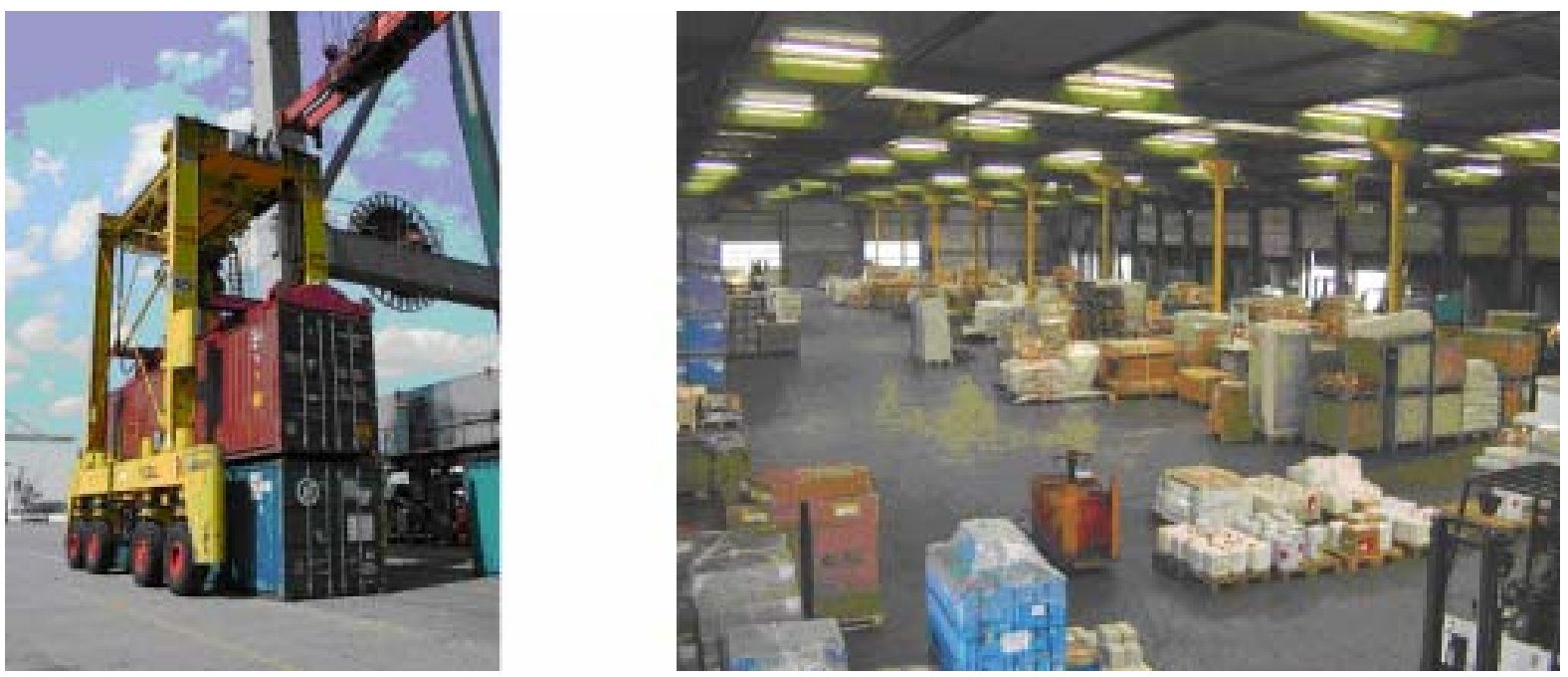

\section{Abbildung 1: Container und Paletten als Ladungsträger}

Dabei ist es wichtig hervorzuheben, dass hiermit nicht nur eine optimale Raumnutzung erreicht wird, sondern dass entsprechende Logistiksysteme (bekanntlich bildet selbst die Transportkette für Übersee-Container keine Ausnahme) auch die gesamte Umschlags- und Handhabungstechnik je nach Ladungsträger abbilden müssen. Auf einzelne Funktionsbereiche bezogen, gilt das also ausnahmslos für die

- Förderprozesse

- Lagerprozesse

- Handhabungsprozesse

Natürlich gibt es neben Containern und Paletten auch „Spezialladungsträger“, wie beispielsweise die Kleinladungsträger (KLT) in der Automobilindustrie oder spezifische Mehrweggebinde für den Handel. Insbesondere die Spezialladungsträger haben im Vergleich zu Paletten und Containern mengenmäßig bei weitem nicht die herausragende Bedeutung, und sie sind darüber hinaus in der Regel abgestimmt auf die Palettengrundmaße der Euro-Palette - wie beispielsweise die KLT. Es handelt sich also um Zwischenladungsträger, die ausgerichtet sind auf das Ziel, mehrere kleine Gebindegrößen zum „Normladungsträger“ zu vereinen. Insgesamt sind die Maschinen und Einrichtungen für die Funktionsschritte „Fördern“, „Lagern“ und „Handhaben“ unter anderem aus den Bereichen Flurförderzeuge und Palettenfördersysteme auf standardisierte Ladungsträger wie Palette und Container abgestimmt.

Auch die inner- und außerbetrieblichen Umschlagsvorgänge sind weitestgehend auf Paletten abgestimmt. Dies gilt sowohl für Distribution, Beschaffung wie auch für produktionslogistische Aufgaben. Die Automatisierung der oben genannten Förder-, Lager- und Handhabungsprozesse erfolgt also in diesem festliegenden Rahmen. Wobei Kommissioniervorgänge, also der Zugriff auf einzelne Waren, in der Regel nur dann automatisiert erfolgen, wenn einfache Produkt-verpackungen oder Produktformen gegeben sind, überdies die Produkte monostrukturiert und in großen Stückzahlen vorliegen. Der automatische Zugriff auf ein einzelnes, durch seine individuelle Geometrie charakterisiertes Produkt ist immer noch weitestgehend ein Wunsch, nicht annähernd schon Wirklichkeit.

\section{Entscheidende Parameter}

Für die Zukunft gibt es zwei sehr wichtige Randparameter, die das Veränderungspotenzial besonders hervorheben:

Die Globalisierung der Weltwirtschaft und der Zwang, aus Kostengründen die eigene Fertigungstiefe zu reduzieren, machen es notwendig, für die Produktentstehung immer mehr Zulieferteile - und damit logistische Prozesse entlang der Supply Chain - zu realisieren. Wie wichtig und weitreichend dieser Sachverhalt ist, erkennt man in Abbildung 2. Dargestellt ist hier, dass die Eigenfertigungstiefe in der deutschen Automobilindustrie bei „nur“ noch $20 \%$ liegt. 


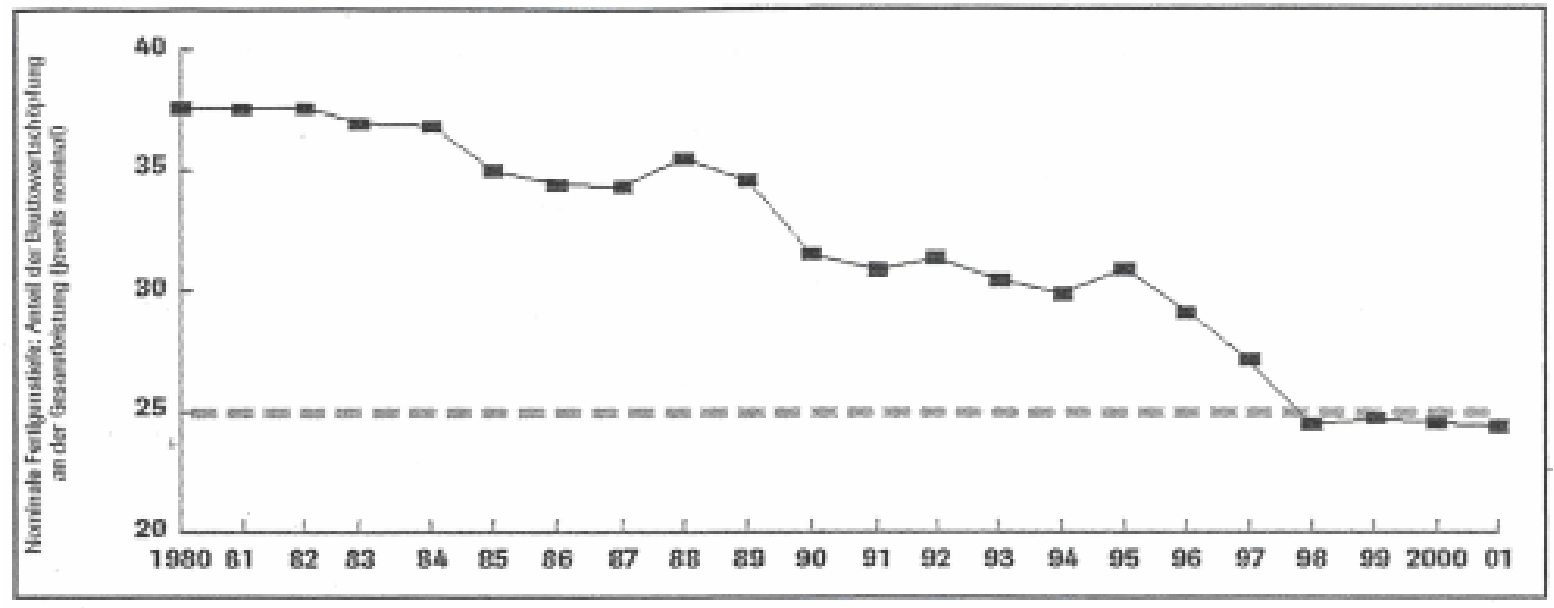

Abbildung 2: Entwicklung der Eigenfertigungstiefe in der Automobilindustrie

Im Bereich der Güter des sogenannten täglichen Bedarfs ${ }^{1}$ sowie in einzelnen Distributionsbereichen, beispielsweise in der KEP-Industrie oder dem Versandhandel, ist eindeutig eine Ladungsatomisierung zu erkennen. Das heißt, immer mehr unterschiedliche Produkte werden angeboten und bestellt, wobei die volumenmäßige Ladungsgröße dieser Produkte ständig abnimmt. Abb. 3 zeigt typische Produkte dieser Art, hier Pakete aus der Kurier-, Express- und Paketdienstbranche sowie aus dem Versandhandel. Deutlich zu erkennen ist, dass diese Sendungen unterhalb der Größe einer Europalette liegen. Selbst wenn man in den Bereich der klassischen Stückgutspedition hineingeht, erkennt man zwar, dass $74 \%$ aller Ladungen palettiert sind (Untersuchung des Instituts für Fördertechnik und Logistik IFT der Universität Stuttgart bei Stückgutspeditionen), dass aber bei diesen Ladungen (entsprechend Abb. 4) eigentlich nur ein Teil der Grundfläche einer Palette benutzt wird und dass die volle Volumennutzung der Palette (800 mal 1200 und 1055mm Beladehöhe) bei weitem nicht erfolgt. Man verwendet hier trotz des geringen Volumennutzungsgrades eine Palette als Ladungsträger, weil die zur Verfügung stehenden Handhabungsmittel - üblicherweise die verschiedensten Arten von Flurförderzeugen, vor allem Handhubwagen und Gabelstapler - wenigstens eine mechanisierte Handhabung der Ladungen zwischen Ein- und Ausgangstor der Speditionszentrale zulassen.

Auch die Volumennutzung der Lkw bzw. Wechselbrücken ist somit unzureichend, was ebenfalls auf die ungenügende Grundvolumennutzung der Paletten zurückzuführen ist.

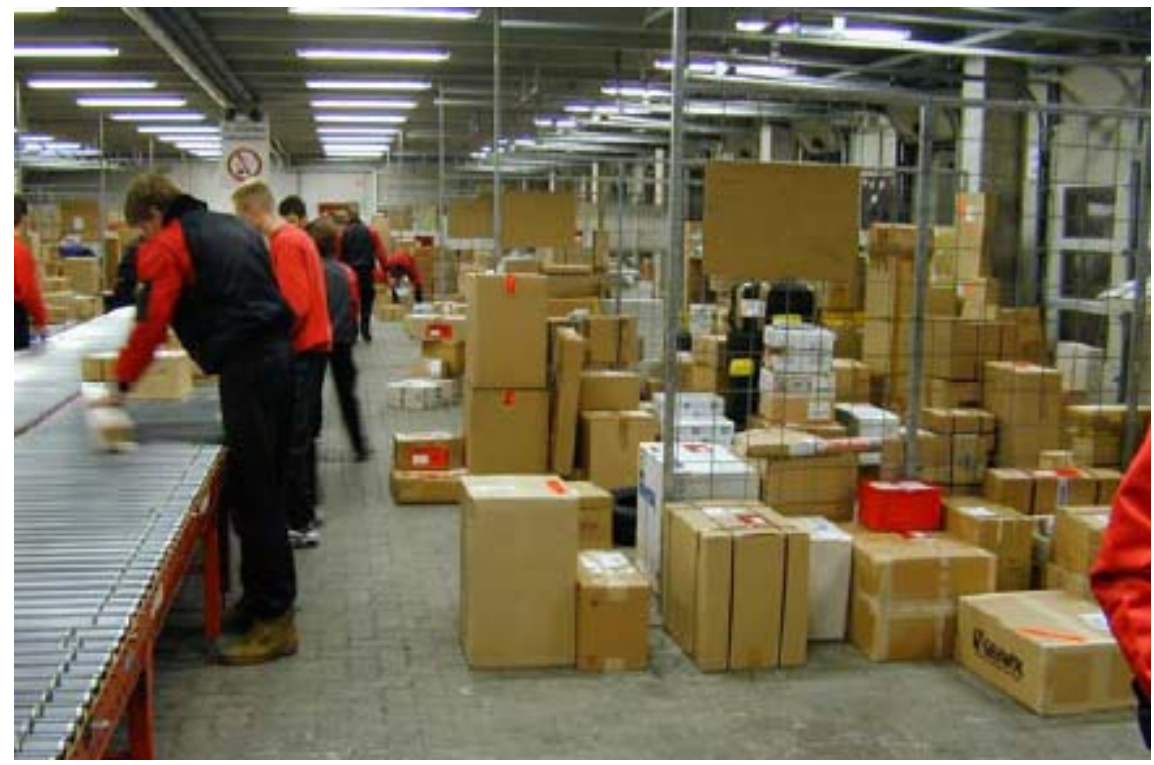

Abbildung 3: Manuelle Paketsortierung am Band

\footnotetext{
${ }^{1}$ Als Güter des täglichen Bedarfs wird ein Produktspektrum verstanden, dass sich aus der heutigen Produktpalette des Versandhandels, des Lebensmittelhandels, des Pharmahandels etc. zusammensetzt. So umfasst ein gut sortierter Versandhandel, z.B. für Winter- und Sommerkollektion, ca. 200.000 Produkte, so dass insgesamt für die Güter des täglichen Bedarfs mit einem Gesamtumfang von ca. 250.000 Produkten als Mindestmenge gerechnet werden muss [Wehking02]
} 


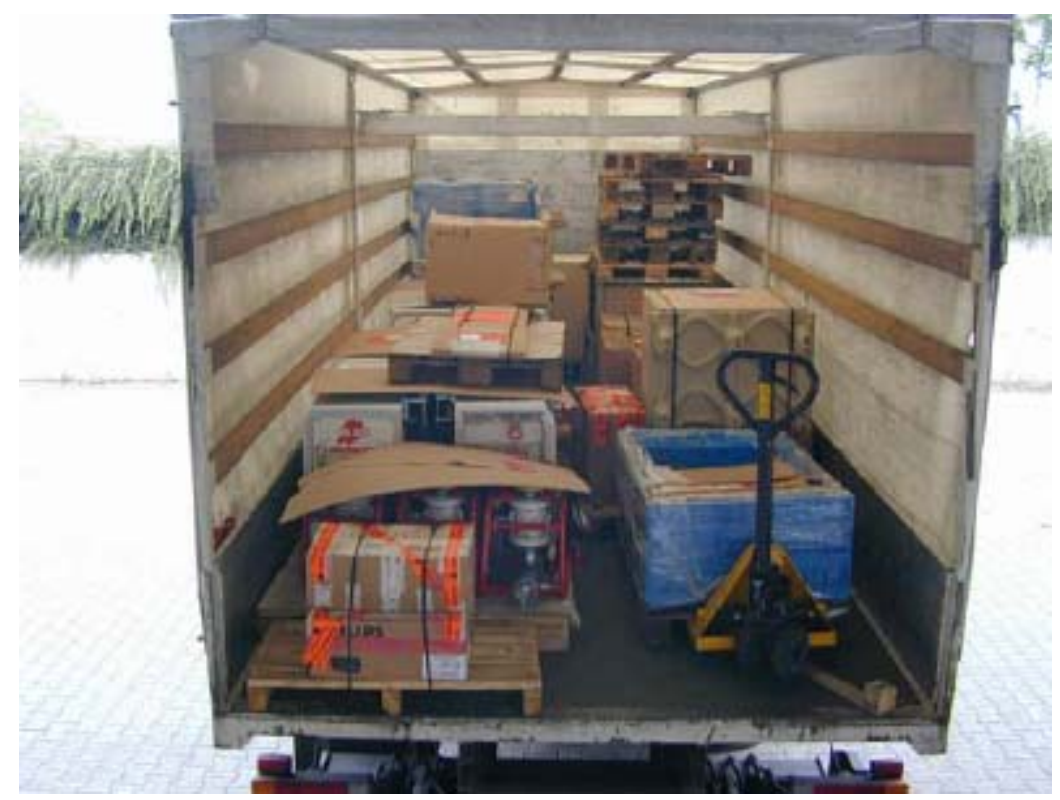

Abbildung 4: Beispiel für unzureichende Volumenausnutzung

Die bisherigen Ausführungen zeigen, dass die heutigen Systeme, z.B. bezüglich der Euro-Palette, den Anforderungen der Zukunft nur unzureichend gerecht werden können. Es kommt hinzu, dass eine wirkliche Automatisierung der Handhabung, vorrangig der Kommissioniervorgänge, bezüglich einzelner Produkte auf dem Ladungsträger, gar nicht oder nur in Ausnahmefällen (monostrukturierte Güter einfachster Form) möglich ist.

\section{Veränderungen erforderlich}

Diese eindeutig erkennbaren Tendenzen werden dann extrem verstärkt, wenn die Dienstleistung der sogenannten E-Logistik, also Internet-gestützte Bestellungen und darauf basierende Auslieferungen bis zum Gemeinschaftsterminal oder Endkundenterminal, tatsächlich im größeren Umfang erfolgt. Derzeitige Schätzungen des Handels besagen, dass dies bis 2006 für etwa 8\% bis 10\% [Forrester01] des stationären Handels erfolgen soll. Das würde ein Gesamtvolumen von geschätzt etwa 100 bis 200 Mio. Euro ausmachen. Die heutige Art der Auslieferung, beispielsweise über Kurierdienste, bei der jedes einzelne Produkt in verpackter Form insgesamt manuell vom Fahrer ,gehandelt“ und vor allem den Kunden übergeben werden muss, lässt für einen Zeitabschnitt innerhalb der nächsten 5 bis 15 Jahre erkennen, dass hier Veränderungen aus wirtschaftlichen Gründen absolut notwendig sind. Diese zwingenden Veränderungen sollen nachfolgend an zwei „realistischvisionären“ Neuentwicklungen im Bereich der Materialflusskomponenten geschildert werden:

\section{Der Bereich der Distributionslogistik}

Die genannte Atomisierung der Ladeeinheit führt in Zukunft zu vielen neuen, kleinen Sendungen, besonders in den Distributionszentren für Güter des täglichen Bedarfs. Die Handhabung erstreckt sich vom Lagereingangsbereich des Distributionszentrums bis zur Anlieferung beim Kunden (Verbraucher-Endterminal oder-Gemeinschaftsterminal).

Da die Produzenten weiterhin ihre Produkte sortenrein auf Basis von Paletten anliefern werden, muss zunächst ein automatisches Lager, hier als Hochregallagerzeile dargestellt, vorhanden sein. Wenn von einem Kunden verschiedene Produkte in der Logistikkette B2C bestellt werden, so sind die verschiedenen sortenreinen Paletten nacheinander zu einem Kommissionierplatz zu befördern, der eine automatische und manuelle Kommissionierung der Waren zulässt.

Die Ware wird von der sortenreinen Palette entnommen und einem neuartigen Ladungsträger, einem sogenannten Tablar, zugeführt. Diese Tablare haben geometrisch modularisierte Größen und sind mit einem RFID-Element versehen. Die gesamte Kundenbestellung, beispielsweise drei oder fünf Produkte, wird auf einen oder mehrere dieser Tablare kommissioniert. Während des Kommissioniervorgangs werden die Daten der Warenbestellung mit der Tablarnummer und den Daten des Endkunden über das RFID-Element, Bestandteil des Tablars, „fest“ verbunden. Angenommen, die Bestellung besteht aus 3 Einzelelementen, dann wird das Tablar (Abb. 5) automatisch in einen modularen Gestellrahmen hineingeschoben. Da die Tablare standardisiert sind, lässt sich hierfür eine Robotertechnik einsetzen. Die modularisierten Gestellrahmen werden anschließend zur Komplettübergabe in ein Auslieferungsfahrzeug bereitgestellt. 
Mit dem Fahrzeug erfolgt anschließend die Anlieferung zum Kunden, wobei der Gestellrahmen mit der fertigen Bestellung über ein mechanisiertes Handhabungshilfsmittel zur Überwindung von Rampen und Treppen direkt zum Endterminal des Kunden oder zu einem Gemeinschaftsterminal, wie beispielsweise dem Tower24, gelangt. Die Abbildung 6 zeigt zusammenfassend die materialflusstechnischen und informations-technischen Komponenten dieses Logistiksystems der Zukunft.

\section{Fern- und Streckentransporte am Beispiel der Bahn}
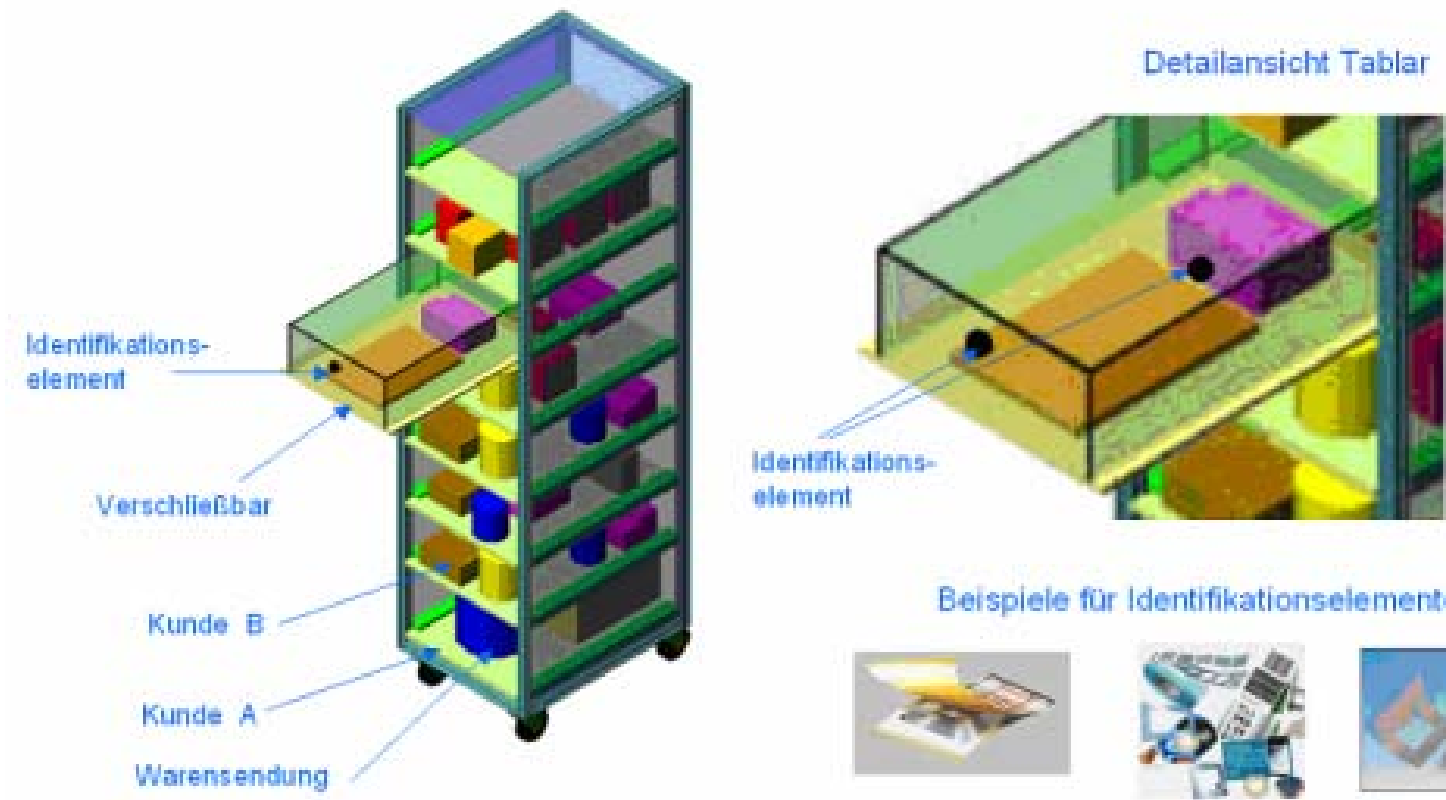

Beispiele für Identifikationselemente:
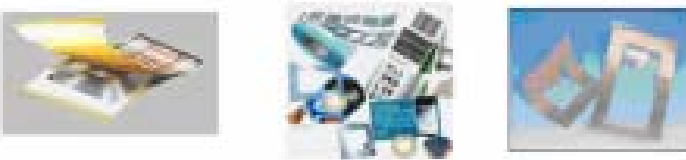

Abbildung 5: Prototyp einer neuen Komponente des E-Business

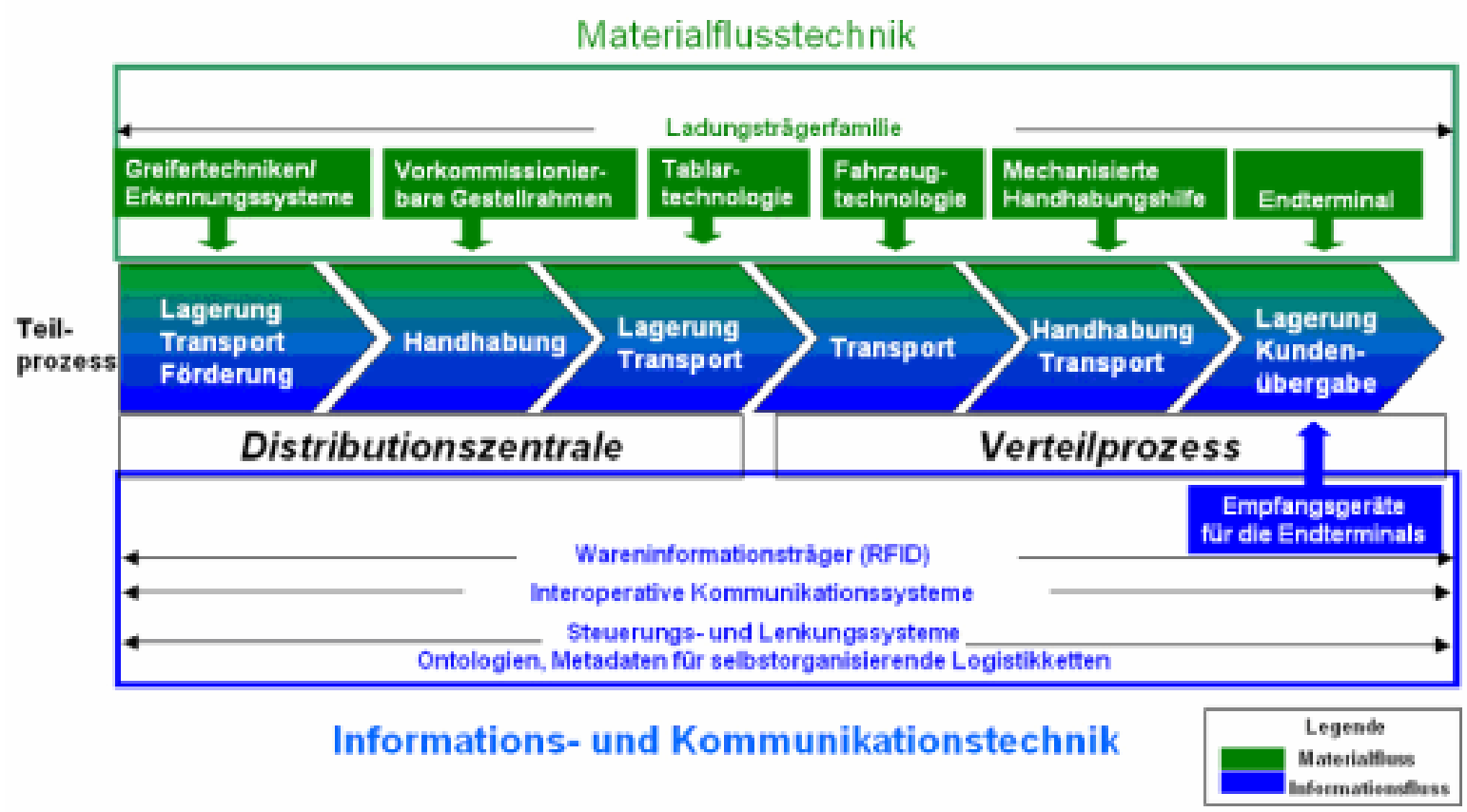

Abbildung 6: Neue Materialflusskomponenten für zukünftige Verteilzentren 
Trotz Privatisierung der Bahn und verschiedener Aktivitäten bleibt die statistische Tendenz ungebrochen, dass die Anzahl der Ferntransporte grundsätzlich steigt, die Menge der im Netz der Bahn transportierten Güter jedoch weiterhin abnimmt (Abb. 7) [BAG99]. Anders formuliert heißt das: Der Straßenferntransport hat die exorbitanten Wachstumswerte des Transportaufkommens vollständig auf seine Seite gezogen.

Trotz verschiedenster Ideen, wie beispielsweise des „kombinierten Verkehrs“ oder der „Ganzzugsverkehre“, ist die Transportmenge der Bahn sowohl im sogenannten Schüttgutbereich als auch im Stückgutbereich rückläufig. Dies gilt vor allem für palettierte Ware, die nicht in ISO-Binnencontainern als Ladungsträger, sondern als Einzelpalette gehandhabt werden muss.

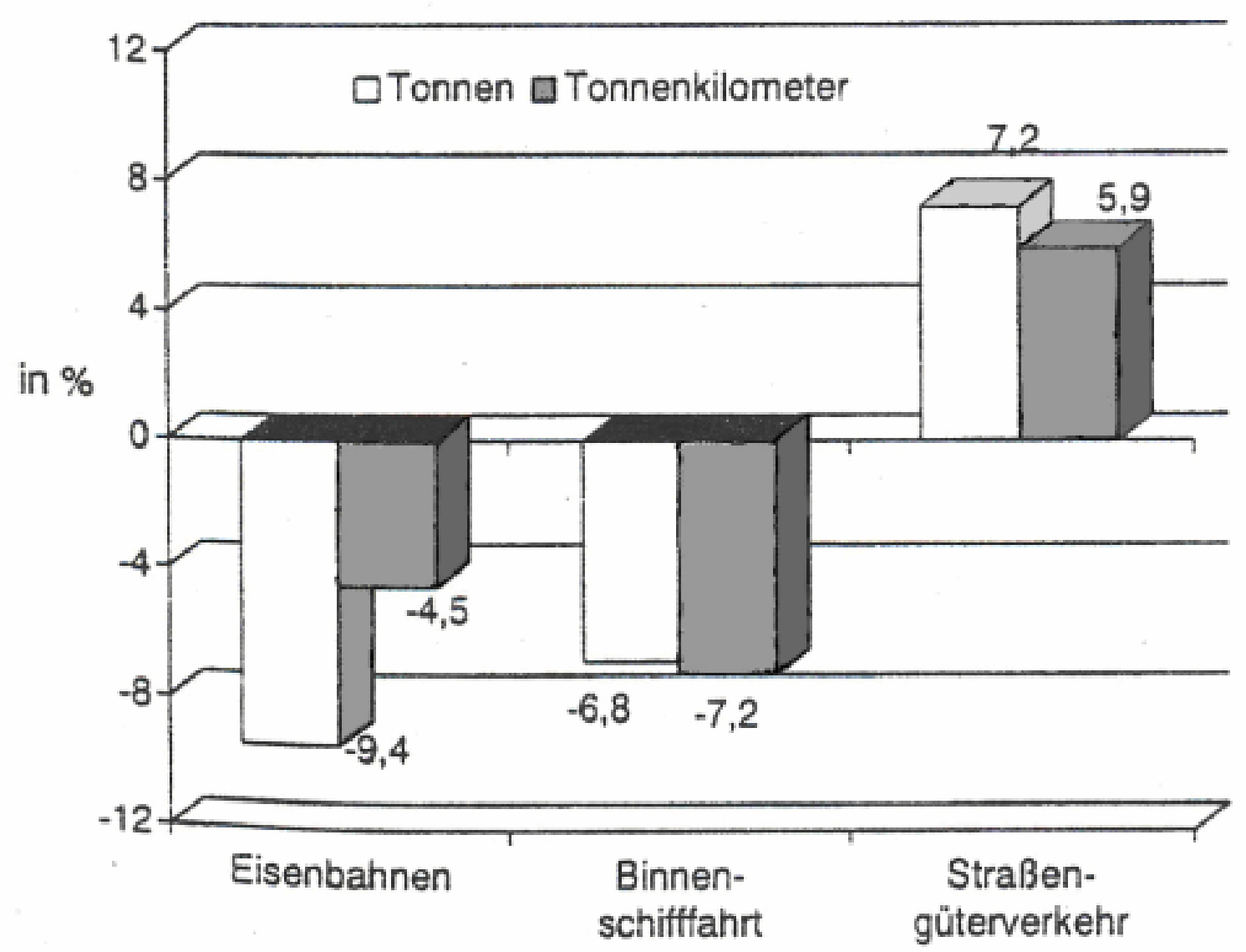

Abbildung 7: Güterverkehr in Deutschland, Veränderung im 1. Halbjahr 1999 gegenüber dem 1. Halbjahr 1998

Die heute grundlegende Bahntechnik, die auf dem Betrieb von Rangierbahnhöfen basiert, lässt wegen der vielen zeitaufwändigen Rangiervorgänge und den damit im Vergleich zum Lkw ungeheuer langen Transportzeiten eine echte Konkurrenzsituation gar nicht erst aufkommen. Zielführend kann hier für die Zukunft nur eine Kombination aus neuer Bahntechnik und neuen, hierauf angepassten Materialflusskomponenten sein.

Auf Seiten der Bahn könnte eine der Lösungen darin bestehen, die im Bereich des Personentransportes eingesetzten Intercity-Netze mit getakteten „Kreuzungs- und Umschlagspunkten“ auch auf den Stückguttransport zu übertragen. Die entsprechende Idee bestünde darin, Nord-Süd- und Ost-WestVerbindungen über die gesamte Republik mit getakteten Ganzzügen, ausschließlich für den Stückguttransport, zu realisieren. Die Ladungsträger müssten an den „Kreuzungs- und Umschlagspunkten (Abb. 8) [Jünemann00] durch entsprechende Technik automatisch von einem auf den nächsten Stückgut-Streckenzug umgeschlagen werden. Die sich im Intercity-Personenverkehr selbstständig bewegenden Personen wären hier (im Stückgutverkehr) durch entsprechende Technik zu ersetzen. 


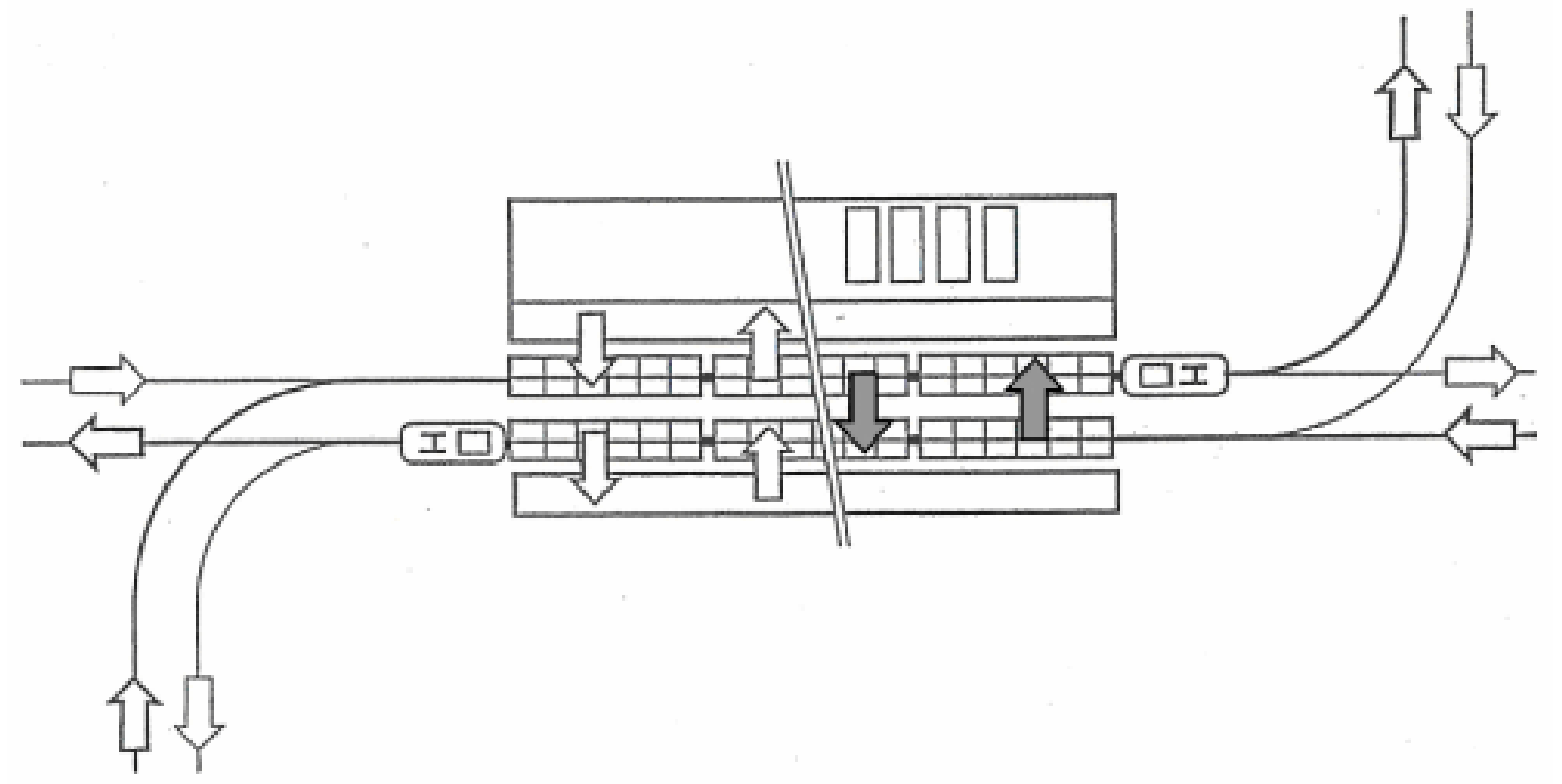

Abbildung 8: Prinzipbild einer Netzstation

Eine im Grunde ähnliche Idee ist bereits 1988/1989 in der damaligen Studie „Cargo 2000“ untersucht worden [Jünemann00]. Hierbei wurden als Ladungsträger spezifische, unter dem Volumenmaß eines DBBinnencontainers liegende neue Kleinladungscontainer (für 2 x 4 Paletten) kreiert. Die oben geschilderte Entwicklung zur weiteren Atomisierung der Sendungen und zu einer weiteren Abnahme der Ladungsgrößen macht es nun nach Ansicht des Autors notwendig, diese Idee konsequenterweise auf Einzelstückgüter und entsprechend kleine Ladungsträger, von der Palette bis hin zu Modul-Racks für Tablare, „,herunterzubrechen“.

Die Abbildung. 9 [Jünemann00] zeigt eine zukünftige Netzstation eines Kreuzungs- und Umschlagspunktes. In der Mitte sind die sich treffenden Züge eingezeichnet. Die Einzelladungsträger auf den Zügen, werden, z.B. durch fahrerlose Transportsysteme, von den Waggons abgeladen und dann entweder direkt auf den wartenden zweiten Zug umgeschlagen oder auf Zwischenpufferbereiche bzw. in ein Lager gebracht. Das Lager dient gleichzeitig zur Anbindung des Straßenverkehrs, mit dem die Feinverteilung der Güter erfolgt.

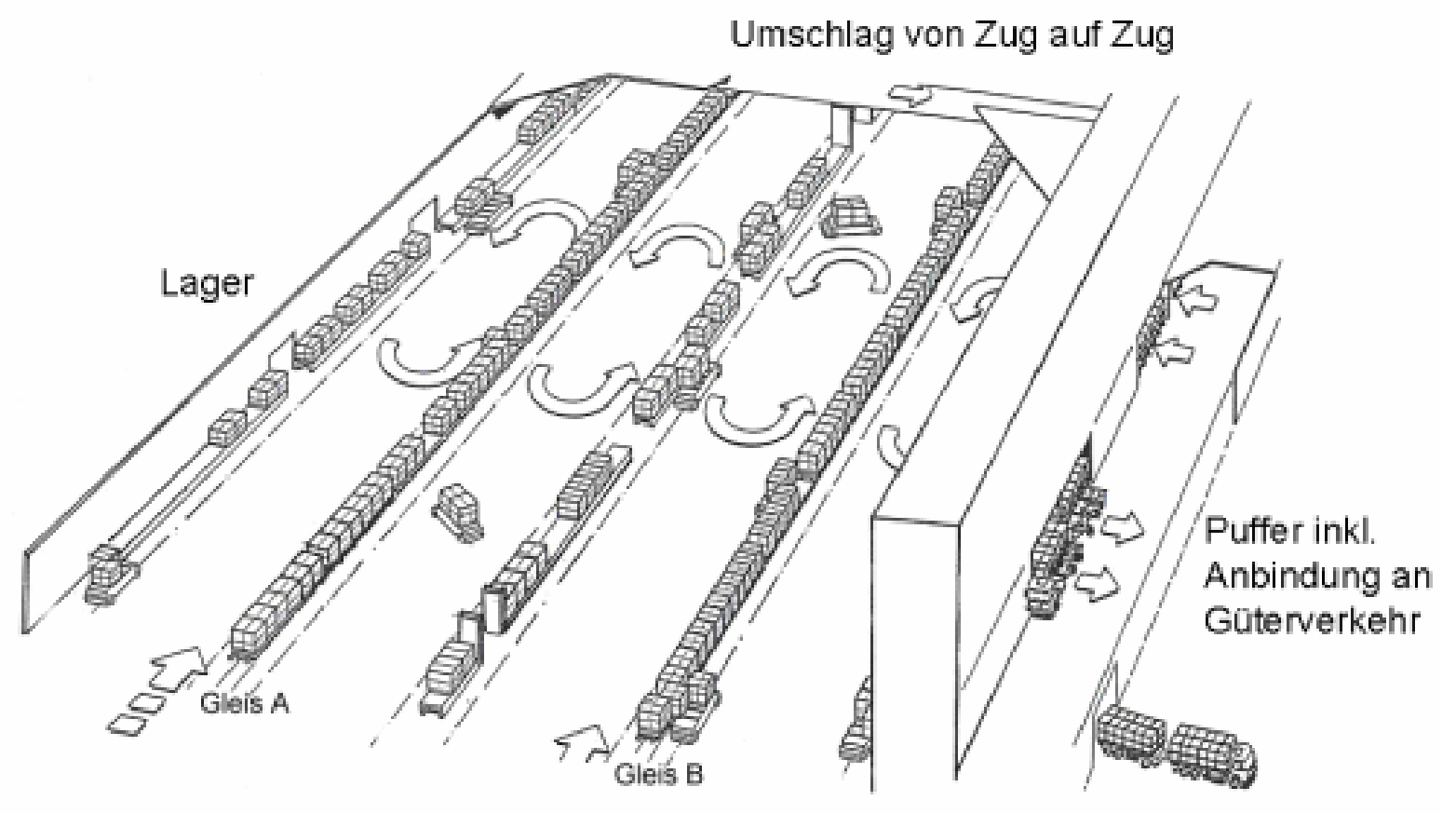

Abbildung 9: Darstellung einer zukünftigen Netzstation 


\section{Zusammenfassung}

Die deutschen Hersteller von Fördertechnik, Materialflusstechnik und logistischen Systemen besitzen aufgrund ihrer langen Tradition eine europa- und weltweite Spitzenstellung in diesem wichtigen Bereich der Wirtschaft. Die oben aufgezeigten Randbedingungen weisen auf zukünftige Herausforderungen und - nach Meinung des Autors - auch auf zukünftige Märkte hin, die durch entsprechend gezielte Entwicklungs- und Forschungsarbeiten „mit Leben“ gefüllt werden könnten, um damit der weltweiten Logistik wichtige Innovationsschübe zu geben. Diese Herausforderung sollte sowohl von der Industrie als auch von der Forschung und Entwicklung an den Hochschulen aufgegriffen werden, weil die hier skizzierten Entwicklungen die besondere Bedeutung der technischen Komponenten zukünftiger Logistiksysteme aufzeigen und damit, gerade in Deutschland, wieder ein zusätzliches zukünftiges Alleinstellungsmerkmal bilden können.

\section{Literatur}

[BAG99]

[Forrester01]

[Jünemann00]

[Klaus]

[Wehking02]
BAG (13109), StBA, KBA, DB AG, VDV, ifo-Institut. In: Marktbeobachtung Güterverkehr, Bericht Herbst 1999, Bundesamt für Güterverkehr Forrester Research: Studie „Europe online retail projects, 2001” http://www.forrester.com Jünemann, Reinhard, Wehking, Karl-Heinz: Cargo 2000 - Jahrbuch des Eisenbahnwesens, 40, S.80 ff. Hestra-Verlag, 1989

Klaus, Peter: Interne Erhebung des IFT, Stuttgart, auf Basis von Daten der Bundesvereinigung für Logistik und des Lehrstuhls für Betriebs-wirtschaftslehre Wehking, Karl-Heinz: Das materielle Internet - zukünftige logistische Strukturen für den E-Commerce bei Business to Consumer-Beziehungen, Jahrbuch der Logistik, 2002

\section{Bildnachweis}

Institut für Fördertechnik und Logistik (IFT) der Universität Stuttgart.

\section{Erstveröffentlichung}

Deutsche Hebe- und Fördertechnik (dhf), Ludwigsburg: A.G.T. Verlag Thum, Ausgabe Juni 2004 